\title{
Pflegekammer:
}

\section{Pflichtmitgliedschaft ist rechtmäßig}

— Die Pflichtmitgliedschaft in einer Pflegekammer entspricht geltendem Recht. Mit dieser Einschätzung hat das Verwaltungsgericht Mainz einer Pflegerin widersprochen, die gegen die Pflichtmitgliedschaft geklagt hatte. Die Klägerin verweigerte zunächst die Übermittlung ihrer beruflichen Meldedaten an den Gründungsausschuss der Kammer, dessen Aufgabe es u.a. war, die beruflich Pflegenden zu registrieren. Mit einer Klage an das Verwaltungsgericht begehrte die Krankenpflegerin sodann die Feststellung, dass sie kein Mitglied der Pflegekammer sei. Sie machte geltend, die Vorschriften des Heilberufsgesetzes, mit denen die Verkammerung von Angestellten in Pflegeberufen geregelt worden seien, verstießen gegen das Grundgesetz; allenfalls eine Mitgliedschaft auf freiwilliger Basis sei rechtlich hinnehmbar. Das Verwaltungsgericht wies die Klage ab. Dem Gericht zufolge ist die Pflichtmitgliedschaft verhältnismäßig, da sie keine elementare Beschränkung der persönlichen Freiheit des einzelnen Mitgliedes darstellt.

„Das ist ein wichtiges und klärendes Signal in der Errichtungsphase der Pflegekammern in Schleswig-Holstein und Niedersachsen. Gleiches gilt für die gerade beginnende Diskussion um eine Pflegekammer in Nordrhein-Westfalen", sagte Martin Dichter, Vorsitzender des DBfK Nordwest. Er hoffe, das Urteil könne zu einer Versachlichung der Diskussion um eine Pflegekammer in NRW beitragen. Eine drängende Frage sei, wie die Pflegenden informiert werden können. Nur so könne sichergestellt werden, dass sich die Berufsgruppe an der Diskussion beteiligt. Zugleich wünscht sich Dichter ein Ende der "Verunsicherung und Fehlinformation der Pflegenden durch Kammergegner hinsichtlich der dauerhaften Darstellung der Pflegekammer als leistungsunfähiges Bürokratiemonster".

www.dbfk.de

\section{Brandenburg plant Umfrage zur Pflegekammer}

- Das brandenburgische Sozialministerium plant eine Informationskampagne zur Pflegekammer. Diese soll das Für und Wider einer Berufskammer für Pflegekräfte beleuchten und noch in der zweiten Jahreshälfte starten. Anschließend sei eine Befragung der Pflegekräfte geplant, teilte das Ministerium der Deutschen Presse-Agentur mit.

Eine Pflegekammer fordern CDU und Grüne in Brandenburg bereits seit langem. Noch im April hatten sich die Oppositionsfraktionen im Landtag für eine solche Interessenvertretung stark gemacht.

www.masgf.de

\section{Hier steht eine Anzeige.}

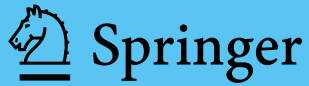

